\title{
Spielformen des Bewusstseins
}

\author{
«Das beflügelte Gehirn», so der Name eines Symposiums, das regelmässig auf dem \\ Monte Verità in Ascona stattfindet. Zahlreiche Referenten äusserten sich zu Phäno- \\ menen und Rätseln des Gehirns, zu Träumen, Formen von Wahrnehmung und Be- \\ wussein, Aussersinnlichem, Wahn und Halluzinationen.
}

Jürg Kesselring

Korrespondenz:

Prof. Dr. med. Jürg Kesselring Rehabilitationszentrum

$\mathrm{CH}-7317$ Valens

Tel. 0813031408

Fax 0813031410

kesselring.klival[at]spin.ch
Die zahlreichen Teilnehmerinnen und Teilnehmer dieses aussergewöhnlichen Kongresses waren zum Teil aus Nebel und Schnee auf den legendären Monte Verità gekommen, wo sie herrlichstes Frühlingswetter mit blühenden Magnolien und weitem Ausblick über den Lago Maggiore erwartete, besonders aber ein vielfältiges Programm von ausführlichen Referaten von ausgewiesenen Experten vor einer interessierten Zuhörerschaft. Spannend (und wohltuend) war die Art der Darstellungen, die alle auf dem Weg gemeinsamen Suchens zeigte, keineswegs einfach als «auf dem Berg der Wahrheit» angekommen.

Im Einfügungsreferat wurde gezeigt, dass die Spielformen des Bewusstseins wohl als Kontinuum aufzufassen sind, das vom Koma (zu dem man auch die Narkose zählen kann) über verschiedene Schlafstadien, unterschiedliche quantitative Stufen und qualitative Färbungen bis zur Wachheit reicht, wobei die Intensität der Weckreize beziehungsweise der Grad des Gewahrseins seiner selbst und der Umwelt als Mass gelten.

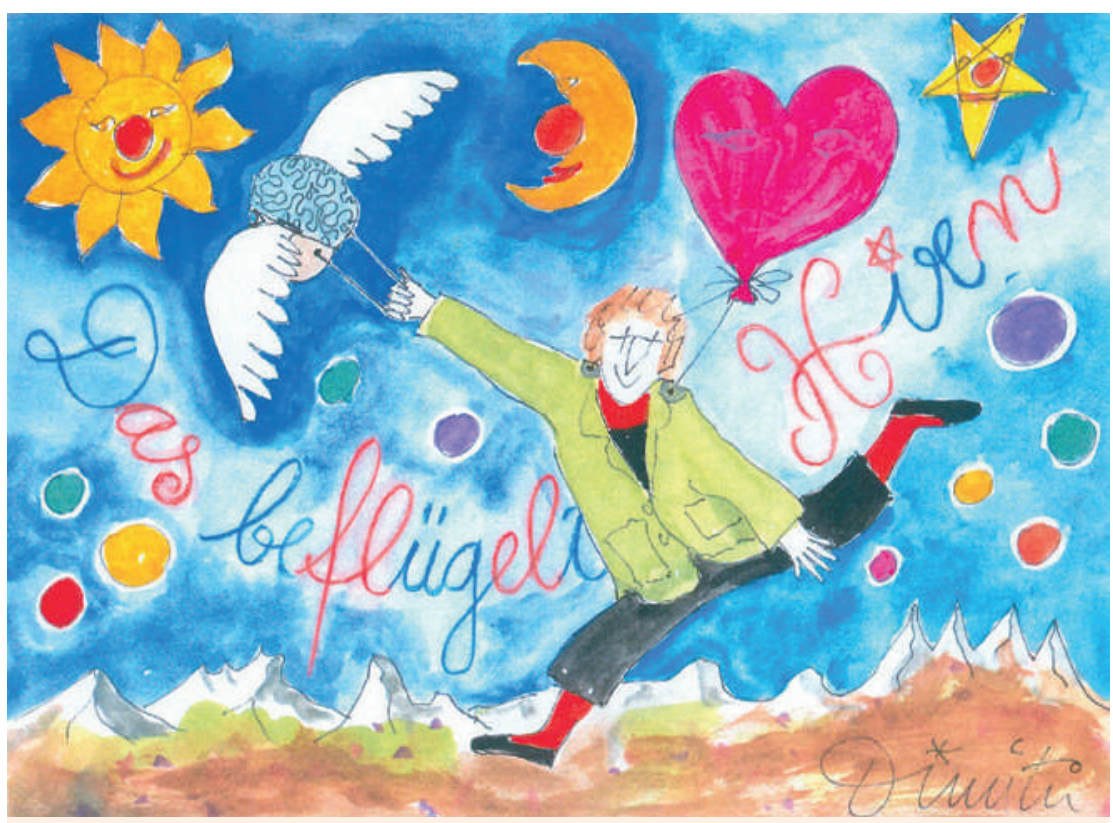

Als Einleitung zu den Vorträgen spielten Studierende der Scuola Dimitri Sketches und Pantomimen, die auf die nachfolgenden Ausführungen verwiesen und diese interpretierten.

Ausgegangen wurde von philosophischen Äusserungen zum Bewusstsein wie von Thomas Huxley (1866): «What consciousness is, we know not; and how it is that anything so remarkable as a state of consciousness comes about as a result of irritating nervous tissue, is just as unaccountable as the appearance of the Djin when Aladin rubbed his lamp in the story» oder der berühmten und pessimistischen Äusserung des Physiologen Emil Du BoisReymond (1831-1889): «Ignoramus et ignorabimus» mit seiner Skepsis gegenüber Erklärungsansprüchen der Naturwissenschaften: «Gegenüber den Rätseln der Körperwelt ist der Naturforscher längst gewöhnt, mit männlicher Entsagung sein 〈Ignoramus` auszusprechen. Im Rückblick auf die durchlaufene siegreiche Bahn trägt ihn dabei das stille Bewusstsein, dass, wo er jetzt nicht weiss, er wenigstens unter Umständen wissen könnte, und dereinst vielleicht wissen wird. Gegenüber dem Rätsel aber, was Materie und Kraft seien, und wie sie zu denken vermögen, muss er ein für allemal zu dem viel schwerer abzugebenden Wahrspruch sich entschliessen: ‘gnorabimus`.» Für den Philosophen Peter Bieri besteht das Rätsel des Bewusstseins darin, dass wir keine Vorstellung davon haben, was als Lösung, als Verstehen zählen würde, es fehlt sozusagen der «readout» der experimentellen Messung und phänomenologischen Erfahrung. Ein Phänomen, ein Sachverhalt ist nur rätselhaft vor dem Hintergrund bestimmter Erwartungen des Erklärens und Verstehens und es kann im Prinzip eine vollständige Kausalerklärung für unser gesamtes waches, integriertes Verhalten beschrieben werden, in der wir als erlebende Subjekte überhaupt nicht vorkommen [1].

Im einleitenden Referat wurden die Agnosien erläutert und die Frage gestellt, wie wir überhaupt dazu kommen, etwas über uns und die Umwelt zu erfahren, ausgehend von den Beschreibungen von Patienten mit Läsionen in verschiedenen Bereichen des Gehirns, besonders der rechten Hemisphäre, die zu so schwer verständlichen, aber klinisch relevanten Erlebnissen wie Neglekt führen, einseitigem nicht Gewahrwerden seines Körpers und der Umwelt und den damit verbundenen schwerwiegenden Einschränkungen in der Alltagsbewältigung. Sorgfältig studierte klinische Bilder können immer noch sehr wohl zu einem besseren Verständnis, auch von phi- 
losophischen Fragen, dienen, aus dem sich dann auch therapeutische Konsequenzen ableiten lassen.

Der Vortrag über Traumbewusstsein von Professor Christian Hess aus Bern begann mit einer sorgfältigen Analyse des berühmten Bildes von Salvador Dalì «Traum, verursacht durch den Flug einer Biene um einen Granatapfel, eine Sekunde vor dem Aufwachen». Nach Rechtsprechung haben wir keine Verantwortung für Taten, die «tatsächlich im Traum» geschehen, subjektiv sind wir aber wohl der Meinung, dass wir von den Traumerlebnissen persönlich angesprochen sind bzw. sie auch selber verursacht haben. Der Traum beschreibt subjektives Erleben, was die Vergleichbarkeit mit Schilderungen anderer Personen erschwert bzw. eigentlich unmöglich macht. Sprache spielt im Traum weniger eine Rolle als Situationen, z. B. auch Farben. Die drei grossen Elemente, die unsere Kultur ausmachen: Lesen, Schreiben, Rechnen kommen im Traum erstaunlicherweise selten direkt vor. Traumbewusstsein ist nahe bei den Halluzinationen. Es gibt Schlafstörungen, die auch in der Malerei (z. B. Johann Heinrich Füssli) grossen Anklang fanden. Der Albdruck bzw. die respiratorische Beklemmung während der Schlafparalyse wird auch heute noch im Volksmund «Toggeli» (= Aufhocker) genannt und findet seine Erklärung im REM-Schlafassoziierten Ausfall der Interkostalatmung. Die Traumsymbolik ist bei Sigmund Freud etwas monoman fixiert auf die Sexualität, während bei C. G. Jung Archetypen und das kollektive Unbewusste eine wichtige Rolle spielen. Im Vergleich mit der Tierwelt ist besonders interessant, wie die für den REM-Schlaf («Traumschlaf») typischen Theta-Rhythmen auch in emotionell beladenen Zuständen vorkommen, etwa bei der Katze beim der Beute Auflauern, beim Kaninchen bei erhöhter Wachsamkeit infolge von Sorgen um die Jungen, bei der Ratte bei Exploration des Umfeldes oder bei uns Menschen beim Hören von Musik. Wegen des amnestischen Effektes des Schlafes erinnern wir nur die Träume beim Erwachen. Typisch sind beim Inhaltlichen Brüche: Kontextdissoziationen, visuelle Skalierungsverschiebungen, Angstzustände. Das alte Dogma, dass Traumschlaf-Entzug zu psychischen und psychotischen Veränderungen führe, wurde nach ausführlichen Nachstudien entkräftigt. Wichtig ist wohl, dass der Traum der Konsolidierung von Gedächtnisinhalten dient. Interessant für Interpretationen ist die Metapher, dass im Traum sozusagen der emotionale Mantel um den Gedächtniskern herum abgebaut wird und diese «Ent-Emotionalisierung» im Schlaf der besseren Rationalisierung und damit besseren Interpretationsmöglichkeiten dient.

Christian Pfeiffer aus Lausanne widmete seine «Magic show» den experimentellen Untersuchungen seiner Forschergruppe zu ausserkörperlichen Wahrnehmungen «out-of-body-experiences» (OBE) und entwickelte (unter Berufung auf den Philosophen Thomas Metzinger, der später zu Wort kam) ein «Minima- les Phänomenales Selbst» mit der Ich-Identifikation («Welcher Körper ist mein?»), der Ich-Lokalisation («Wo bin ich im Raum?») und der Erste-Person-Perspektive, die krankhaft dissoziiert sein können bei autoskopischen Halluzinationen, Heautoskopie und Doppelgängertum, und eben OBEs. Diese können auch manipuliert werden durch Aktivierung im temporoparietalen Übergang der rechten Hirnhemisphäre.

\section{Wie kommen wir dazu, etwas über uns und die Umwelt zu erfahren?}

Die Psychosomatikerin Verena Kesselring aus der Rehabilitationsklinik Valens setzte ihre Ausführungen zu den dissoziativen Gangstörungen unter den Titel «Ich will gehen, aber es geht nicht», womit sie gleichsam Freuds grosses Modell von Über-Ich, Ich und Es in einer Nussschale zusammenfasste und aus zahlreichen, sorgfältig ausgewählten historischen Texten und Beispielen aus der klinischen Tätigkeit überzeugend illustrierte. Sie betonte, dass psychische ursächliche Faktoren und Komorbiditäten oft vorliegen, aber bei 20-50\% der Betroffenen auch mit sorgfältiger psychiatrischer Exploration nicht nachgewiesen werden können. Neurophysiologische Untersuchungen zeigen einen Unterschied zwischen Simulation und dissoziativen Bewegungsstörungen und stimmen überein mit mathematischen Modellen, die einen Mismatch von Bewegungsvorhersage (-erwartung) und proprioceptiver Rückmeldung auf verschiedenen neuronalen Ebenen dafür verantwortlich machen, dass die Bewegungsstörung als unwillkürlich empfunden wird und nicht korrigiert werden kann. Die feine und im Alltag, besonders auch bei Gutachten, so wichtige Unterscheidung von dissoziativen Störungen und echter (seltener) Simulation kann eher gelingen, wenn erfasst werden kann, wie sich die Erwartungen an eine Bewegung und die Rückmeldung von ihrem Erfolg unterscheiden können. Es ist ja auch in unserem Leben leider nicht so selten der Fall, dass Erwartung und Erfolg nicht übereinstimmen und dann so oft irgendeiner aussenstehenden Macht eine Schuld zugeschoben wird.

Der besonders originelle Neuropsychologe Peter Brugger widmete sich mit sorgfältiger Sprachanalyse und experimentellen Befunden den «aussersinnlichen Wahrnehmungen» (ASW), wobei er das Kürzel wohl besser als «Ausdruck sequentieller Wahrscheinlichkeit» interpretierte. Mittels Letzterem konnte er sehr einleuchtend zeigen, wie z. B. telepathische Übertragungen auf naturwissenschaftlichen Gesetzen basieren. $\mathrm{Ob}$ die beiden miteinander kommunizierenden Medien sich auf zwei verschiedenen Kontinenten oder in zwei getrennten Räumen eines Hauses befinden, spielt dabei keine Rolle. 
Der Philosoph Thomas Metzinger aus Mainz ist mit seinen Publikationen und Vortragstätigkeiten ein ausgewiesener Kenner der Bewusstseinsphilosophie und gab auch in seinem Vortrag zahlreiche Belege für seine Kenntnisse, die auch praktisch umsetzbar sind (und sein sollten), etwa wenn er den Widerspruch bzw. die Deckungsungleichheit von bewusstem und unbewusstem Selbstmodell als (Mit-)Ursache für psychosomatische Störungen erkennt. Bei der Verkörperung unterscheidet er die prä-rationale Intelligenz, fragt anhand von eindrücklichen Roboterbeispielen, ob ein Ich oder ein Selbst denn eine Illusion sei und widerlegt dann aber eine solche Frage als «unsinnig», weil ja eine Illusion ein wissendes Selbst voraussetze, und ein Erleben nicht schon Wissen sei.

Eindrücklich und emotional gefärbt waren die Einleitungen zu den jeweiligen Vorträgen durch Studierende der Scuola Dimitri, die in brillant gespielten Sketches und Pantomimen auf die nachfolgenden theoretischen Ausführungen verwiesen und diese im Voraus interpretierten.

Der Abend des ersten Symposiumstages ermöglichte gemütliches Zusammensein und Gedankenaustausch von Referenten und Teilnehmerinnen im Teatro Dimitri in Verscio. Dort wurde von Nina Dimitri und Silvana Gargiolu ein grossartiges «Concerto Rumoristico» gegeben, wo die wunderbaren, kräftigen und differenzierten Stimmen in bolivianischen Volkliedern und gekonnt-humoristischen Einlagen genossen werden konnten.

Der zweite Tag stellte in seiner Frühlingspracht den ersten noch in den Schatten und führte den schönsten Bewusstseinsaspekt: die Besonnenheit vor die Augen und ins Gemüt. Thomas Reber entwickelte ein differenziertes, experimentell gut abgestütztes Modell zur Rolle des Hippokampus bei Gedächtnisfunktionen und beim Lernen, die für ein besseres Verständnis von Bewusstsein so wichtig sind. Unterstrichen wurde die Rolle des präfrontalen Kortex bei der «Interpretation von Bedeutung».

Ein ganz praktischer und von Vielen gefürchteter, deshalb enorm wichtiger Aspekt des Bewusstseins wurde von Thomas Heidegger diskutiert: «Awareness» während der Anästhesie, die Angst davor, während der Narkose aufzuwachen, d.h. Bewusstsein zu erlangen, bzw. (etwas weniger verbreitet!), dass dies danach nicht mehr gelingen könnte. Da nimmt man dankbar in Kauf, dass das Augenöffnen als vielleicht einfaches Mass für Bewusstsein gewählt wird, wenn man dann vorgeführt bekommt, mit welcher praktischen Kenntnis den verschiedenen Störmöglichkeiten nachgegangen wird, damit sie vermieden werden können, und mit welcher Sorgfalt und psychologischem Gespür auf die Patienten eingegangen wird, wenn diese sich dann doch einmal über ein solches Vorkommnis äussern.

Der Direktor der Psychiatrischen Universitätsklinik Waldau in Bern, Prof. Werner Strik, sprach über das gleichzeitig erschreckende wie faszinierende Phä- nomen des Wahns als Ich-Störung, die (ausser bei der seltenen «folie-à-deux») immer einsam ist und macht. Das Bewusstsein der Persönlichkeit hat immer die Möglichkeit zur Ich-Entwicklung zur Voraussetzung und deshalb beruht es eigentlich auf einer Selbsttäuschung, wenn man meint, man sei zu jedem Zeitpunkt immer genau dieselbe Person: «Man nimmt eigentlich nicht sich selber wahr, sondern diejenige Persönlichkeit, die sie vor wenigen Augenblicken war» (Carl Wernicke). Bewusstsein ist eine «höhere Hirnfunktion» und deshalb nicht lokalisierbar - eine passende Metapher dafür wäre, dass man die Geschwindigkeit eines Autos auch nicht in der Analyse seiner Bestandteile verstehen kann. Zum Selbst-Bewusstsein gehört ein «Sense of agency» und ein «Sense of ownership». Halluzinationen kommen im Wahn typischerweise vor und sind gleichsam «laute Gedanken von aussen». Neurophysiologisch interessant ist der Nachweis (der natürlich nur selten gelingt), dass bei Halluzinationen eine gleichzeitige Stimulation von Hörrinde und Sprach-Kortex nachgewiesen werden kann. Wahn ist eine unverrückbare, inhaltlich falsche Überzeugung, die nicht Teil einer Subkultur ist, und ist charakterisiert durch einen Drang, sich auszudrücken, ein Empathiedefizit und eine mehr oder weniger ausgeprägte Kampfbereitschaft. Die Psychose ist eigentlich eine grundlegende Störung der Kommunikation, bei der falsche Informationen (Trugwahrnehmung), logische Fehler (Inkohärenz) und eine starke Affektspannung («Sprung zu Schlussfolgerungen») zusammenlaufen. Sie ist eigentlich immer mit Angst vergesellschaftet, die sich in verschiedenen Weisen der Kommunikation und des Verhaltens äussert: Erstarrung (Mutismus, Hilfesuchen usw.), Flucht (Ausweichen, Nachgeben, Weglaufen) oder Kampf (Widerspruch, Anschuldigung, Drohung, Beleidigung usw.).

Der Psychologe Walter Perrig aus Bern zeigte die Entwicklung des Selbstbewusstseins zunächst in der Entwicklung des Kindes, das erst allmählich im Alter von 2-3 Jahren ein Vergangenheitsbewusstsein ausbildet (und deshalb sind kaum je Erinnerungen an die Zeit davor möglich), während zuvor ein Prozesserleben dominiert, das auch bei uns Erwachsenen wichtig bleibt. In raffinierten Experimenten mit «eingebetteten» Bildern oder «maskierten «Wörtern (und Non-Wörtern) lassen sich Bewusstseinszustände differenzieren, die unterschiedlich unser Verhalten im Alltag beeinflussen. Wohltuend ist die Einsicht, dass eine gewisse Leichtigkeit, eine Geläufigkeit und Vertrautheit eines Prozesses das Charakteristikum von Meisterschaft eines Verhaltens ist: eine wissenschaftlich fundierte Aussage zum Grundgefühl, das der Tagung den Namen gegeben hat: «Das beflügelte Gehirn».

\section{Literatur}

1 Bieri P. Was macht Bewusstsein zu einem Rätsel? In: Metzinger Th (Hrsg.). Bewusstsein. Beiträge aus der Gegenwartsphilosophie. Paderborn: Schöningh; 1995. 